\title{
A Detail Survey of Channel Access Method for Cognitive Radio Network (CRN) Applications toward 4G
}

\author{
Shivam, $\mathrm{Y}^{1^{*}}$, Prashant, $\mathrm{K}^{2}$, Ravi, K. $\mathrm{S}^{3}$, Vivek, $\mathrm{R}^{4}$, Devasis, $\mathrm{P}^{5}$ \\ ${ }_{1,2,3,4}$ Final Year U.G Students, Department of Electronics \& Communication Engineering, Acharya Institute of \\ Technology, Bengaluru - 560107, Karnataka, India \\ ${ }^{5}$ Assistant Professor, Department of Electronics \& Communication Engineering, Acharya Institute of Technology, \\ Bengaluru -560107, Karnataka, India
}

\section{*Corresponding Author \\ Shivam, Y}

\section{Article History}

Received: 25.01.2021

Accepted: 09.02.2021

Published: 16.02.2021

\begin{abstract}
A number of latest technologies are being integrated by means of the telecommunications enterprise because it prepares for the subsequent technology cellular offerings. One of the key changes integrated within the multiple channel get entry to strategies is the choice of Orthogonal Frequency Division Multiple Access (OFDMA) for the air interface. This paper affords a survey of various a couple of channel get right of entry to schemes for $4 \mathrm{G}$ networks and explains the significance of these schemes for the development of spectral efficiencies of cognitive radio network. The paper also discusses approximately using a Multiple Input and Multiple Output (MIMO) strategies to improve sign reception and to combat the results of multi-path fading. A comparative overall performance analysis of different multiple access schemes inclusive of Time division multiple access (TDMA), FDMA, Code division multiple access (CDMA) \& Orthogonal frequency division multiple access (OFDMA) is made vis-à-vis layout parameters to focus on the advantage and limitations of these schemes.
\end{abstract}

Keywords: Cognitive Radio, CRN, Energy, FDMA, TDMA, CDMA, OFDMA, MIMO.

\section{INTRODUCTION}

The Federal Communications commission FCC has costumed the spectrum frequencies to apply it for one use, so while this frequency certain for one person meaning we are in risk of going for walks out of spectrum and awful utilization of it [1]. In keeping with that the FCC defines a cognitive radio networks CRN to solve that problem. The definition of cognitive radio states that it's a radio that able to alter its transmitter parameters primarily based on interaction with its operation environment [2]. CRN permits to dynamically using of spectrum, a CRN became referred to as cognitive radio community [3]. Cognitive radio CR is an a improved to software defined Radio (SDR) that its routinely detects the encircling $\mathrm{RF}$, catalysts and smartly incorporates its running parameters to the infrastructure of community in line with meet consumer demand [4], if this band is further used by a certified person, the cognitive radio stirs to other spectrum band or stays within the identical band with changing its degree of the transmission energy or modulation scheme all of that ward off interference, calibrations the congestion because of spectrum participating [4].

The primary features for cognitive radios in $4 \mathrm{G}$ networks can be summarized as follows;

a) Spectrum Sensing: spotting utilized spectrum and sharing the spectrum without deprived interjecting with different Customers.

b) Spectrum Control: fascinating the fine available spectrum to meet person communication demands [5]

c) Spectrum Mobility: preserving tractable communication exigencies all through transferring to higher spectrum [6].

d) Spectrum Sharing: offering an equitably spectrum scheduling approach between cohabitation 4G customers [7].

Copyright (C) 2021 The Author(s): This is an open-access article distributed under the terms of the Creative Commons Attribution 4.0 International License (CC BY-NC 4.0) which permits unrestricted use, distribution, and reproduction in any medium for non-commercial use provided the original author and source are credited. 
Cognitive radio networking is a new concept in wireless communication in which the network or cognitive radio themselves changes their parameters to do their task efficiently without disturbing the other cognitive radio node. The cognitive radio system therefore used to define the efficient use of spectrum by allowing the Unlicensed user (secondary user) SU to access the spectrum allocated to licensed user (primary user) PU. The channel efficiency is given as ratio of data rate and bandwidth.

$$
\text { Efficiency }=\frac{\text { Data Rate }}{\text { Bandwidth }}
$$

\section{Cognitive Radio}

In modern day radio structures aren't privy to their radio spectrum environment as they may be designed to perform in a predefined frequency band the use of a selected spectrum access device. As discussed in the creation, maximum of the time the spectrum is not correctly applied. The spectrum utilization can be advanced appreciably by permitting secondary users (unlicensed) to dynamically get right of entry to spectrum holes that is temporally unoccupied with the aid of the number one user as shown in Fig-1.1

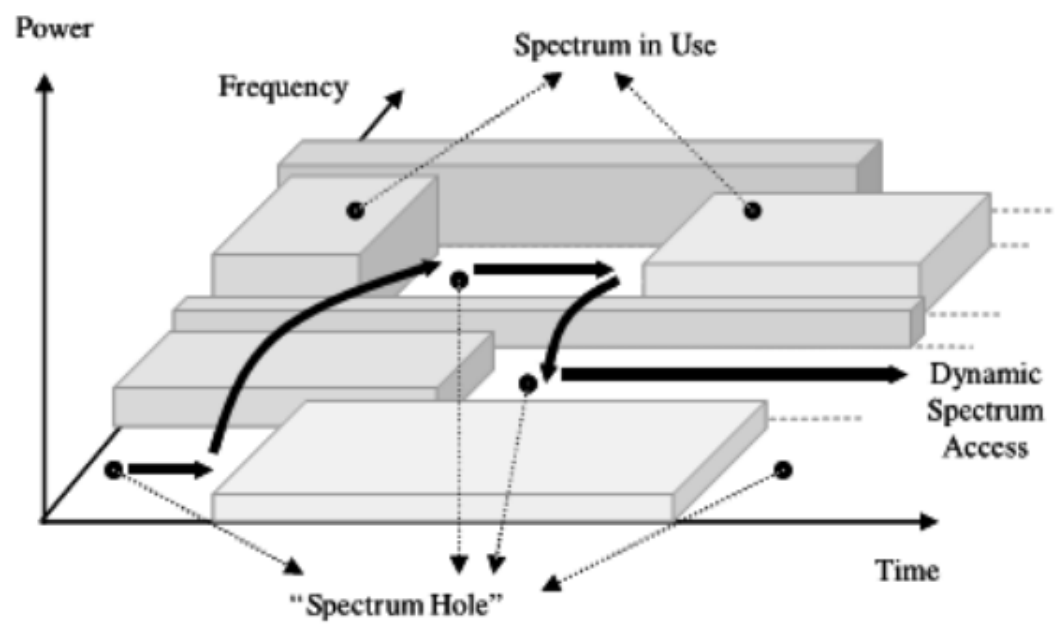

Fig-1.1: Spectrum Hole Concept

To improve the present day spectrum underutilization mental characteristic Radio systems has been visible as a promising solution whereas the increasing amount of services and applications in wireless networks [6] is accommodating. With an identical band due to the element to at an equal time or opportunistically function mental characteristic radio era could probably maximum probably modify accomplice diploma whole SU system. But, as outcomes of form of demanding situations it faces in but it learns the occasion of mental characteristic radio continues to be at a abstract level and at every stop of the hyperlink adapts to the native spectral activity. To acknowledge their communication placing the inherent characteristic of those chemical element structures would be their capability and for the SUs while minimizing the interference to maximize the quality of service (QoS) to the PUs adapts the parameters of their verbal exchange subject matter. [14-15].

However, in channel excellent and interference accordingly on beat high variation metal systems should be forced to possess a high diploma of flexibility. In SDR era as a effects of implicit realization of these characteristics it is going to be designed over bundle made public radio (SDR), that's already in production and is currently on hand of the radio putting (in phrases of spectrum utilization, energy spectral density of transmitted/acquired indicators), dynamic capability (adaptive standardization to device parameters like transmit electricity, service frequency, modulation strategy and so on.) and intensely low-budget cooperative or non-cooperative behaviour (while there's opposition among a couple of steel transceivers), the important thing picks of steel transceivers location unit consciousness. The Figure 1.2 shows the cognitive cycle. 


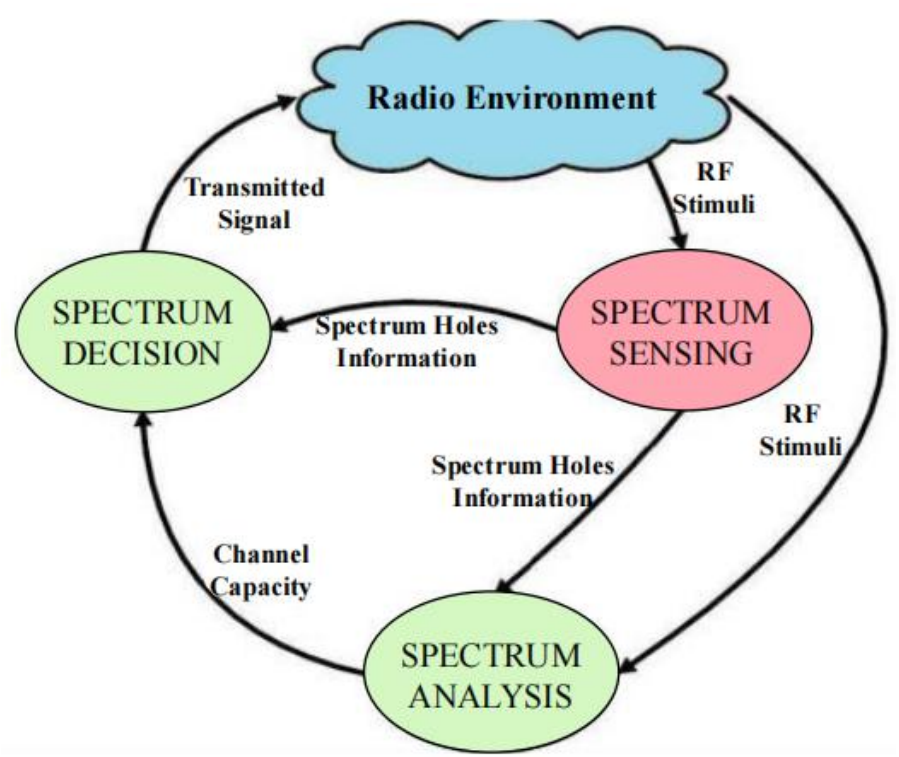

Fig-1.2: Cognitive Cycle

For a CR community to be deployed for s mart usage type of new technology must be pressured to be advanced. For particular hobby region unit the challenges worried the various layout of bodily and hyperlink layers. For network parameters, dependable spectrum sensing (detecting international enterprise used spectrum), spectrum satisfactory (preserving seamless transition to a replacement spectrum), being (with PUs and absolutely exceptional metallic networks), spectrum management, irresponsible (in phrases of QoS), aid allocation (including transmit electricity allocation and dynamic spectrum sharing (DSS)) thus on region unit kind of new mechanisms among those layers Like live, should be compelled to be designed for several budget friendly and a long way harmless get entry to and sharing of expedient spectrum. Moreover, to spice up community performance for various things thus as it's crucial to fine optimize these mechanisms.

\section{Cognitive Re-Configurability}

Re-configurability is the caliber of adjusting the parameters of working for the transmission at the fly without changing at the hardware components. This ability allows the CR to dynamically version with the radio environment [10]. There are numerous reconfigurable parameters that can be protected into the cognitive radio as defined beneath.

a) Operating frequency; In Keeping With Ability of CR to Trade Its Working Frequency. Based On The Radio Environment Statistics, The Most Suitable Running Frequency May Be Determined And The Verbal Exchange Can Be Dynamically Officiated On This Suitable Running Frequency.

b) Modulation; In Keeping With Channel Situations And Person Necessities The Modulation Scheme Of CR Ought To Be Adaptive [10].

c) Transmission Power; Power Constraints Are Manage The Transmission Energy Reconfiguration By Permitting Dynamic Configuration For Transmission Power Within The Permissible Strength Restrict. If Higher Energy Operation Isn't Important, The CR Reduces The Transmitter Power To A Decrease Level To Decrease The Interference And Permit Greater Customers To Percentage The Spectrum [9].

d) Communication Technology; Amongst Special Communication Systems. Cognitive Radio Used To Enable Interoperability. The Transmission Parameters Of A Cognitive Radio Can Be Reconfigured For The Duration Of The Transmission [7].

Consistent with the spectrum traits those parameters may be reconfigured such that the cognitive radio is switched to a one of a kind spectrum band, the parameters of the transmitter and receiver are reconfigured and the perfect modulation schemes and communique protocol parameters are used.

\section{Cognitive Radio System Architecture (CRSA)}

There are three major components inside the cognitive radio community as;

a) Spectrum authorities; Those Are the Spectrum Right Proprietors, Such As Authorities Who Offers the Right to the Community Operators for Long Term Use.

b) Primary Networks: Those Are Number One Base Stations and Number One Quit Users.

c) Secondary Networks: The Secondary Base Stations Dynamically Request And Access Available Spectrum Of PU. 
For the usage of the spectrum of PU, SU make payments to primary users as an incentive for the number one users to borrow the licensed spectrum. "A Cognitive radio is a system that senses its operational electromagnetic surroundings and can dynamically and autonomously adjust its radio operating parameters to modify gadget operation, which includes maximize throughput, mitigate interference, facilitate interoperability, get admission to secondary markets. [6-8]

The simple cognitive radio network structure [4] is proven in determine 5 which shows that spectrum authority gives permission to the network operators (primary customers) to apply the spectrum for an extended duration .Special secondary customers (known as cognitive user) senses the spectrum that the PU is transmitting or no longer. Relying on availability that if now not then SU sends request to secondary base station to apply the spectrum of PU simplest if it isn't transmitting. The architecture shown in Fig-1.1

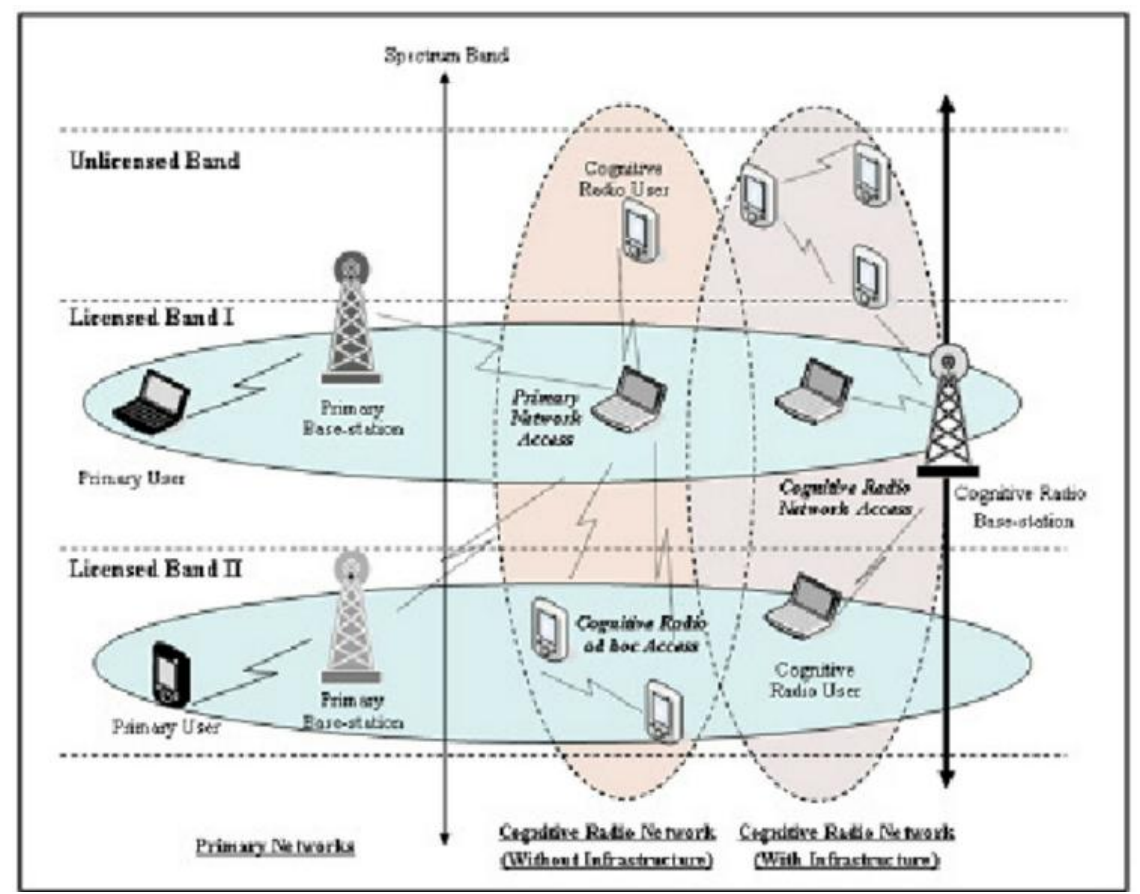

Fig-2.1: Cognitive Radio System Architecture

\section{G Network}

According to the $4 \mathrm{G}$ operating businesses, the infrastructure and the terminals of $4 \mathrm{G}$ may have nearly all of the standards from $2 \mathrm{G}$ to $4 \mathrm{G}$ carried out. Despite the fact that legacy systems are in place to undertake current users, the infrastructure for $4 \mathrm{G}$ may be best packet-based totally (all-IP). Some proposals advise having an open net platform. With the wireless requirements evolution, the get entry to techniques used additionally increased in efficiency, capability and scalability. The primary generation wireless standards used undeniable Time division a couple of get right of entry to (TDMA) and Frequency division more than one get entry to (FDMA). Inside the wireless channels, TDMA is less efficient in managing the excessive information charge channels because it calls for large guard periods to alleviate the multi path impact. Again, FDMA consumes more bandwidth for guards for keeping off inter service interference. So two branches got here in second era structures, one branch of trendy used the mixture of FDMA and TDMA and the opposite delivered a new get admission to scheme called Code division multiple access (CDMA) [8-9].

Usage of CDMA expanded the system capacity. Facts rate is likewise extended as CDMA is efficient to deal with the multi path channel. This enabled the 1/3 technology structures to use CDMA as the get right of entry to scheme of IS-2000, UMTS, HSXPA, 1xEVDO, TD-CDMA and TD-SCDMA. The best difficulty with CDMA is that it suffers from poor spectrum flexibility and scalability. Currently, new get right of entry to schemes like Orthogonal Frequency division a couple of get entry to (OFDMA), Single Carrier FDMA (SC-FDMA), Interleaved FDMA (IFDMA) and MultiCarrier CDMA (MC-CDMA) are gaining greater importance for the subsequent era structures. WiMax makes use of OFDMA within the downlink and in the uplink [9]. For the subsequent technology UMTS, OFDMA is being considered for the downlink. Through contrast, IFDMA is being considered for the uplink considering OFDMA contributes extra to the peak-to-average energy ratio (PAPR) associated troubles and results in nonlinear operation of amplifiers. 
IFDMA provides much less electricity fluctuation and as a consequence avoids amplifier problems. Further, MC-CDMA is inside the thought for the IEEE 802.20 standard. This is an introduced advantage in particular within the MIMO environments because the spatial multiplexing transmission of MIMO systems requires excessive complexity equalization on the receiver. With these benefits in those multiplexing systems, improved modulation techniques are also used. At the existing statistics quotes of $15-30 \mathrm{Mbit} / \mathrm{s}, 4 \mathrm{G}$ is able to imparting customers with streaming high-definition television. At rates of one hundred Mbit/s, the content material of a DVD-5 (as an example a film), can be downloaded within about five mins for offline access [10-12].

\section{Role of Cognitive Radio on 4G}

While fully carried out, $4 \mathrm{G}$ is predicted to enable pervasive computing, wherein simultaneous connections to a couple of high speed networks provide seamless handoffs during a geographical place. The network Operators may additionally rent technology together with cognitive radio and wi-fi mesh networks to make certain connectivity and efficiently distribute both visitors and spectrum [13-15]. Multiple requirements of $3 \mathrm{G}$ make it difficult to roam and interoperate throughout diverse networks, whereas $4 \mathrm{G}$ gives worldwide preferred that gives global mobility. This is viable with the assist of cognitive radio.

As a guide of Mobility management the conversation between distinct systems ought to be hooked up thru regular interfaces. Multi-mode terminals are the one factor taken into consideration for $4 \mathrm{G}$ structures. 4G systems will show to be a ways cheaper than $3 \mathrm{G}$, considering they may be constructed atop existing networks and won't require providers to buy pricey more spectrum.In addition to being lots greater value green, so vendors can do greater with less [12]. With $4 \mathrm{G}$ systems there might be a need to layout a unmarried person terminal which could perform in unique wireless networks and triumph over the design issues inclusive of limitations in size of the tool, its fee and electricity intake.

This problem may be solved the usage of software program defined Radio/Cognitive Radio method i.e consumer terminal adapts itself to the Wi-Fi interfaces of the community. Any other essential function of Cognitive Radio in 4G communications is that the 4G gadgets are expected to be extra visible and intuitive in place of these days's textual content and menu primarily based systems. They may be capable of engage with the environment around it and act for that reason. [16-17]

\section{Multiple Access Schemes for 4G Mobile Wireless Networks (Frequency Division Multiple Access FDMA)}

FDMA offers users an character allocation of one or numerous frequency bands, or channels. It is a primary technology within the analog advanced mobile phone carrier (AMPS), the maximum extensively-mounted mobile smartphone device installed in North America. With FDMA, each channel may be assigned to most effective one user at a time. FDMA is also used in the overall get admission to communication machine (TACS). The virtual-superior cell phone provider (D-AMPS) additionally makes use of FDMA but provides TDMA to get 3 channels for every FDMA channel, tripling the number of calls that can be treated on a channel. The use of frequency division multiplexing is to offer a couple of and simultaneous transmissions to a unmarried transponder. Figure 3.1 shows FDMA

\section{Frequency Division Multiple Access}

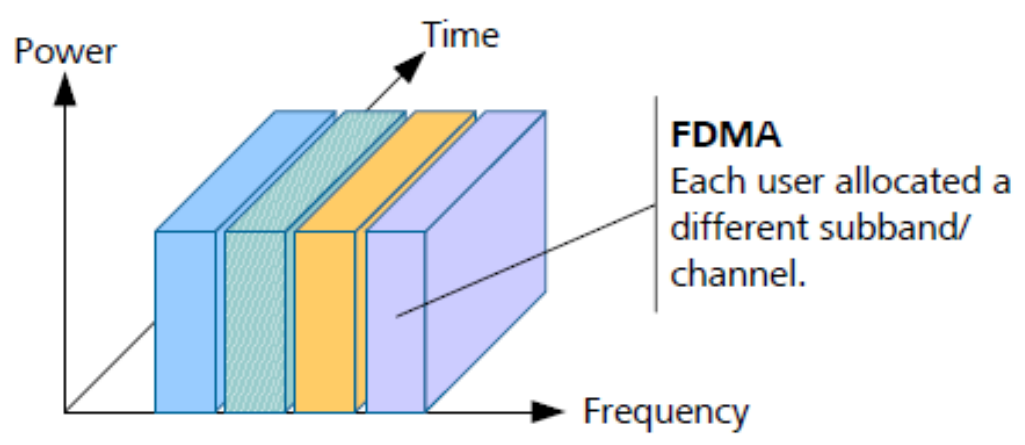

Fig-3.1: Frequency Division Multiple Access (FDMA)

In FDMA, every transmitter is assigned a wonderful frequency channel in order that receivers can discriminate amongst them by way of tuning to the favored channel. TDMA and CDMA are usually used in combination with FDMA, i.e., a given frequency channel may be used for both TDMA or CDMA independently of indicators on other frequency channels. In 1989, the mobile Telecommunications enterprise affiliation (CTIA) chose TDMA over Motorola's FDMA (today referred to as narrowband analog mobile-phone carrier [NAMPS]) narrowband widespread as the era of choice for 
existing $800 \mathrm{MHz}$ cellular markets and for rising $1.9 \mathrm{GHz}$ markets. Crosstalk is a first-rate limitation of FDMA, which reasons interference between the other frequency bands and disturbs the transmission.

The functions of FDMA are as follows.

(i) FDMA calls for high-performing filters inside the radio hardware, in contrast to TDMA and CDMA.

(ii) FDMA isn't at risk of timing issues like TDMA. Due to the fact that a predetermined frequency band is available

(iii) For the whole duration of communication, stream data can without problems be used with FDMA.

(iv) As frequency filtering is there, FDMA isn't always sensitive to close to-a long way problem which we get in CDMA.

(v) There is distinct frequency slot for each user transmission and reception takes place at one of a kind frequencies.

There is a difference among FDMA and frequency-division duplexing (FDD). Whilst FDMA allows more than one users to concurrently get entry to a certain device, FDD refers to how the radio channel is shared among the uplink and downlink (as an example, the traffic going from side to side among a telephone and a basestation). Again, Frequency Division Multiplexing (FDM) is different from FDMA. FDM is a bodily layer method that mixes and transmits low bandwidth channels via a excessive-bandwidth channel. FDMA, alternatively, is an access method inside the information link layer [11]. Low PAPR and occasional sensitivity to service frequency offset are some of the beneficial residences of FDMA. A hybrid device can be fashioned by way of combining TDMA and FDMA.

\section{Time Division Multiple Access}

TDMA is a channel get admission to method for shared medium networks. It permits several users to share the same frequency channel through dividing the sign into special time slots. The users transmit in rapid succession, one after the alternative, each at his time slot. This permits a couple of stations to proportion the same transmission medium (e.G. Radio frequency channel) even as using simplest a part of its channel capacity. TDMA is used in the virtual $2 \mathrm{G}$ cell structures which includes international device for cellular Communications (GSM), IS-136, private virtual mobile (\%) and iDEN, and in the Digital Enhanced Cordless Telecommunications (DECT) popular for transportable telephones. It is also used extensively in satellite structures, and combat-net radio structures. [19]

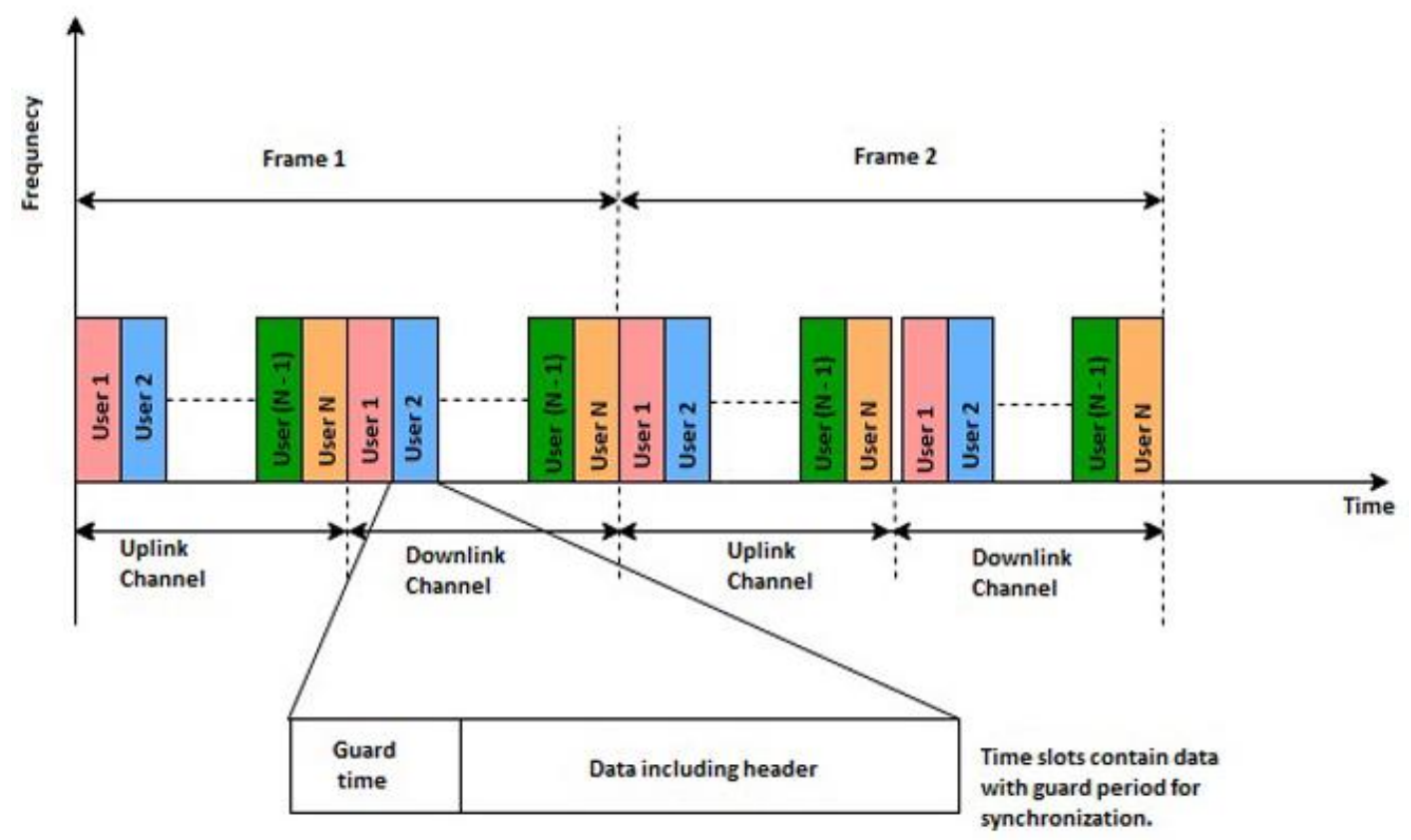

Fig-3.2: Time Division Multiple Access (TDMA)

TDMA is a method used to enable more than one earth stations or VSAT terminals to transmit intermittently on the identical frequency, however with the timing in their transmissions so arranged that the bursts do not overlay whilst they come on the satellite but arrive in series and for this reason are all successfully acquired by the teleport hub modem burst demodulator. The operation of TDMA requires an out link manage to all the far off websites which contains a few control statistics. This out link provider also had a frame structure that offers accurate timing facts for all of the far flung sites. [19-20].

The teleport hub equipment pc tells each VSAT site what particular time slot to apply within the TDMA body and this time plan information is broadcast to all websites periodically. The burst time plan can be fixed, in an effort to allocate every website online a particular share of the whole TDMA frame time or is may be dynamic, whereby the time 
slot allotted is adjusted in response to the traffic desires of each website [12]. Figure 3.2 indicates the TDMA frame structure where a statistics circulation is split into frames and those frames divided into time slots.

TDMA is a form of time-division multiplexing, with the special point that rather of having one transmitter connected to 1 receiver, there are a couple of transmitters. In the case of the uplink from a mobile smartphone to a base station this turns into in particular difficult because the cellular cellphone can flow around and range the timing strengthen required to ke its transmission in shape the distance in transmission from its peers.

The functions of TDMA are as follows;

a) Shares single provider frequency with a couple of customers

b) Non-non-stop transmission makes handoff simpler

c) Slots can be assigned on demand in dynamic TDMA

d) Less stringent electricity manipulate than CDMA due to decreased intra mobile interference

e) Higher synchronization overhead than CDMA

f) Superior equalization can be necessary for excessive information quotes if the channel is "frequency selective" and creates Inter symbol interference

g) Cell respiration (borrowing resources from adjoining cells) is more complex than in CDMA

h) Frequency/slot allocation complexity

i) Pulsating electricity envelop: Interference with other gadgets

\section{Code Division Multiple Access}

CDMA employs spread-spectrum generation and a unique coding scheme (where every transmitter is assigned a code) to allow a couple of users to be multiplexed over the identical bodily channel. By evaluation, TDMA divides access by means of time, while FDMA divides it with the aid of frequency. CDMA is a form of "spread spectrum" signaling, for the reason that modulated coded sign has a much higher data bandwidth than the facts being communicated. CDMA has been extensively used in mobile telephones and in satellite TV for PC device for transportation logistics. The scheme of CDMA is briefly defined underneath. CDMA makes use of the spread spectrum approach, which spreads the bandwidth of the statistics uniformly for the equal transmitted energy. Spreading code is a pseudo-random code which has a slender ambiguity characteristic not like other slender pulse codes.

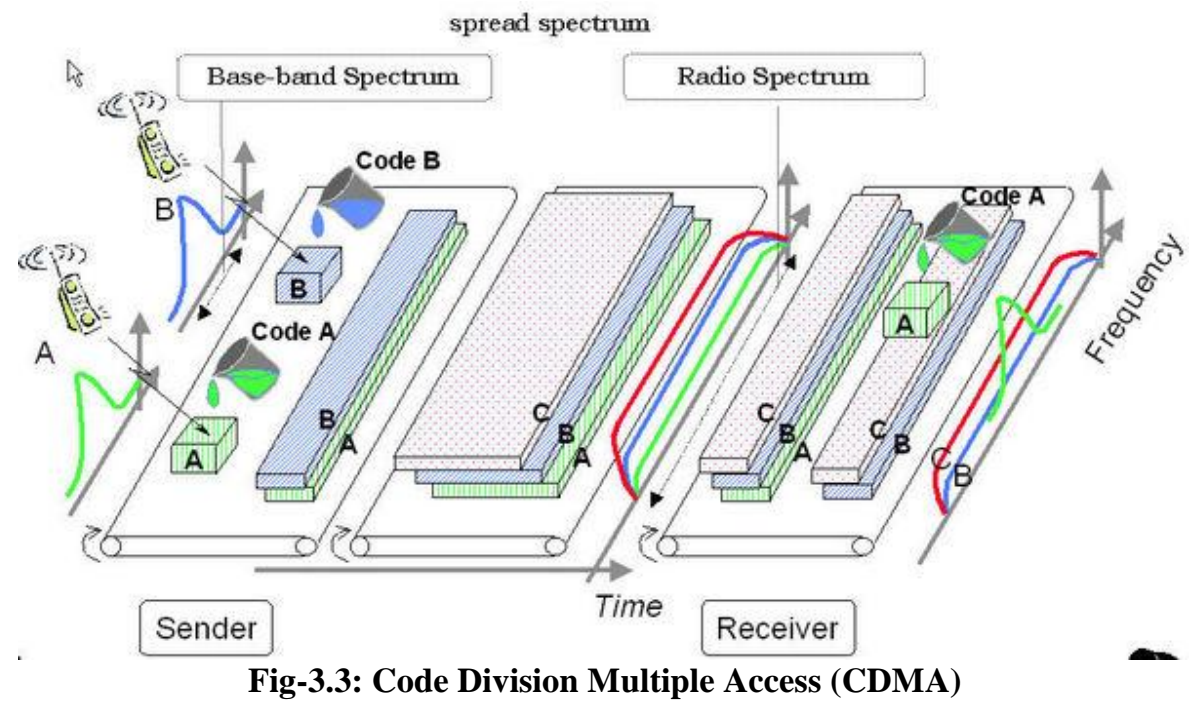

In CDMA a domestically generated code runs at a far higher rate than the facts to be transmitted. Statistics for transmission is simply logically XOR (special OR) brought with the faster code. The parent indicates how unfold spectrum signal is generated. The facts sign with pulse duration of Tb is XOR introduced with the code sign with pulse period of Tc. (observe: bandwidth is proportional to one / $\mathrm{T}$ wherein $\mathrm{T}=$ bit time) therefore, the bandwidth of the statistics signal is $1 / \mathrm{Tb}$ and the bandwidth of the spread spectrum signal is $1 / \mathrm{Tc}$. Due to the fact that Tc is an awful lot smaller than $\mathrm{Tb}$, the bandwidth of the spread spectrum signal is lots larger than the bandwidth of the authentic sign. The ratio $\mathrm{Tb} / \mathrm{Tc}$ is referred to as spreading factor or processing advantage and determines to positive extent the upper limit of total variety of users supported simultaneously with the aid of a base station. Fig. 2 suggests the body shape of CDMA. Every user in a CDMA gadget uses a distinct code to modulate their signal. Deciding on the codes used to modulate the signal may be very crucial in the performance of CDMA systems. The best overall performance will arise when there is ideal separation among the sign of a favored consumer and the alerts of other customers. 
The separation of the signals is made by correlating the received sign with the regionally generated code of the desired consumer. If the sign fits the preferred person's code then the correlation function might be excessive and the machine can extract that sign. If the desired user's code has nothing in common with the sign the correlation must be as close to zero as feasible (for that reason removing the signal); this is known as move correlation. If the code is correlated with the sign at any time offset aside from 0,the correlation have to be as close to zero as possible. That is called carcorrelation and is used to reject multi-route interference. Flexible allocation of assets and private protection in due to anti-jamming competencies of PN sequences are some of the advantages of CDMA. [8-9]

\section{Orthogonal Frequency Division Multiple Access (OFDMA)}

OFDMA is a multi-person version of the famous OFDM virtual modulation scheme. For attaining multiple accesses subsets of sub-carriers are provided to the man or woman customers in OFDMA. This lets in simultaneous low statistics charge transmission from numerous customers. OFDMA is also a candidate get entry to method for the IEEE 802.22 wireless regional area networks (WRAN). The assignment pursuits at designing the first cognitive radio based totally preferred running within the VHF-low UHF spectrum (television spectrum) [15]. FDMA additionally supports call for challenge with constant project. Demand venture allows all customers reputedly non-stop get right of entry to of the radio spectrum by assigning carrier frequencies on a temporary foundation the usage of a statistical challenge technique. Single carrier - FDMA (SC-FDMA) is a form of FDMA scheme, which is basically a multi-user version of Single-Carrier Frequency-Domain-Equalization (SC-FDE) modulation scheme [16]. Figure 3.4 shows OFDM signals.

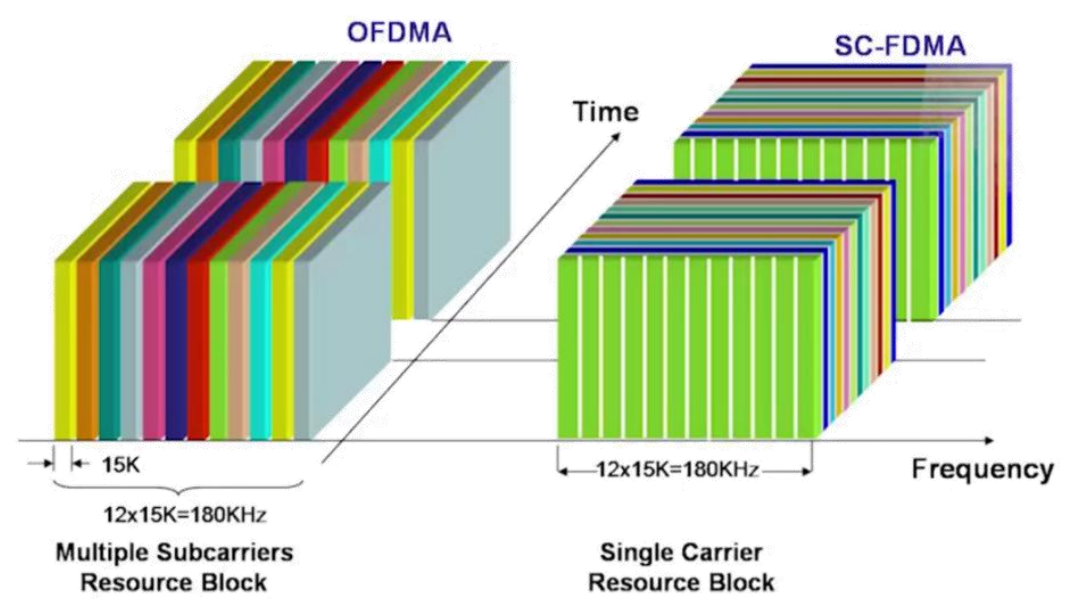

Fig-3.4: Frequency time representation of OFDMA vs SC-FDMA signal

Based totally on comments data approximately the channel conditions, adaptive user-to-subcarrier task may be accomplished. If the undertaking is done sufficiently speedy, this in addition improves the OFDM robustness to fast fading and slim-band co channel interference, and makes it possible to acquire even higher device spectral efficiency. Extraordinary number of sub-vendors may be assigned to one-of-a-kind users, for helping extraordinary great of provider (QoS), i.e. to control the records charge and errors opportunity individually for each consumer. OFDMA resembles CDMA unfold spectrum, wherein customers can reap one-of-a-kind information rate through assigning a extraordinary code spreading element or a specific wide variety of spreading codes to all users. OFDMA can be visible as an opportunity to combine OFDM with TDMA or time-domain statistical multiplexing, i.e. packet mode data exchange. Low data-rate users can send continuously with low transmission energy rather than the usage of a "pulsed" excessivepower provider. Consistent delay, and shorter delay, may be achieved. In OFDMA, the assets are partitioned inside the time frequency area, and slots are assigned alongside the OFDM symbol index in addition to OFDM sub-service index. OFDMA is taken into consideration as relatively suitable for broadband wi-fi networks, due to blessings including scalability and MIMO friendliness, and ability to take gain of channel frequency selectivity. In spectrum sensing cognitive radio, OFDMA is a possible technique to filling loose radio frequency bands adaptively.[9-10] 


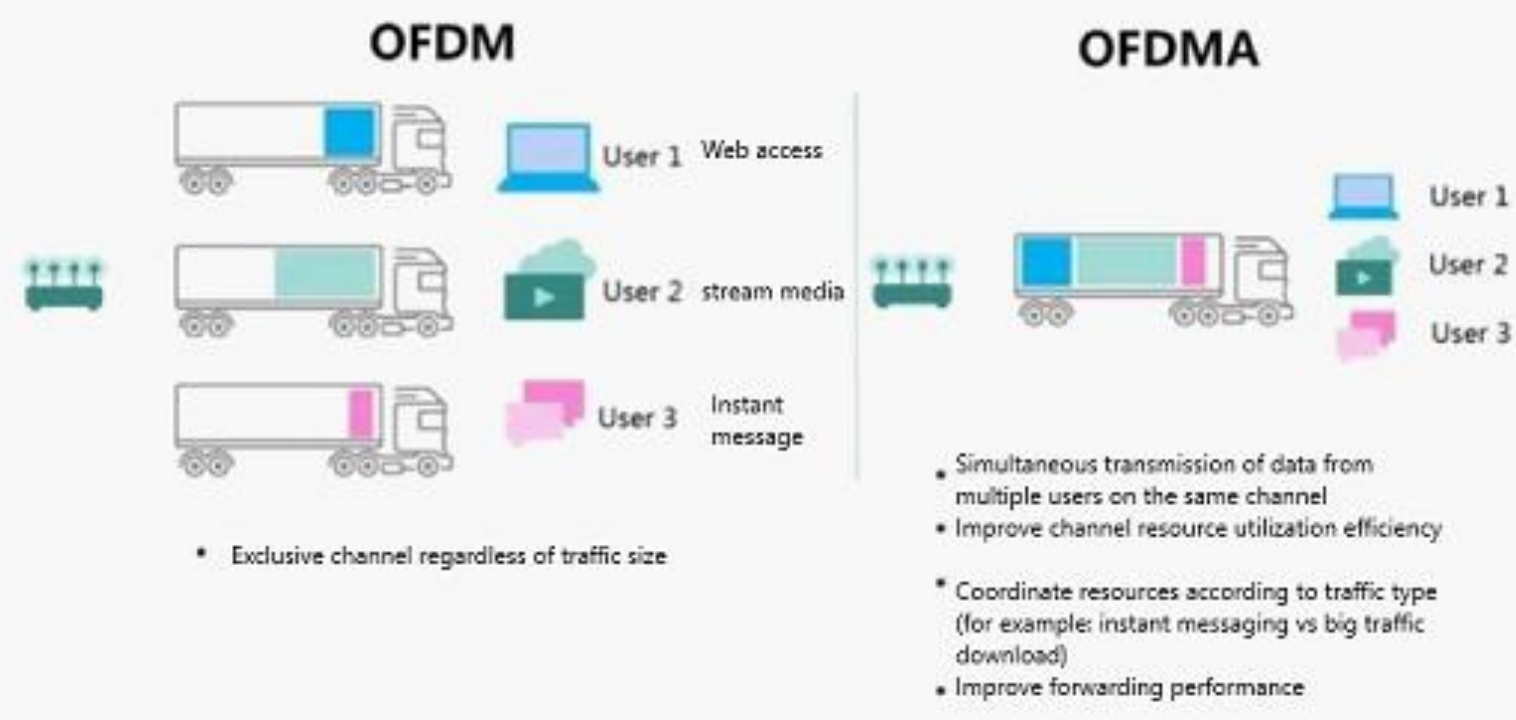

Fig-3.5: OFDM vs OFDMA (Source: Huawei.com)

\section{MIMO - OFDMA}

Cognitive radio (CR) in conjunction with MIMO and orthogonal frequency-division multiple access(MIMOOFDMA) is a candidate era for destiny mobile radio networks. The quick verbal exchange range of underlay $\mathrm{CR}$ structures is typically a first-rate proscribing element. On this paper, had endorse a computationally and spectral green resource allocation scheme for multi-user MIMO-OFDM based totally underlay CR networks to provide precise spectral performance benefit, and therefore multiplied communique variety. The scheme is foremost for the down link however,however, near-highest quality for the uplink. [17-18].

In this paper had compare the overall performance of MIMOOFDM Cognitive radio device where CR devices constantly experience the channel to test whether or not it's miles idle or no longer using compressed sensing with cyclostationary detection and reconstruct the sign if communication is for the given CR receiver from its transmitter. We use the opportunity of mis- detection and opportunity of fake alarm as metrics to evaluate the spectrum sensing, MSE, and successful reconstruction price (SRR) as metics to assess the reconstruction of the sign for CR conversation [19].

On the equal time, as a clever spectrum sharing technology, Cognitive Radio (CR) became additionally proposed to decorate the utilization of the spectrum utilization. Accordingly, the aggregate of MIMO-OFDM and Cognitive Radio, MIMO-OFDM based totally Cognitive Radio era is treated as a prospect scheme for future dynamic spectrum get admission to community or spectrum sharing gadget. Given that simplest a finite wide variety of subcarriers are occupied by means of the number one customers (PUs) in CR networks, the secondary users (SUs) can discover the spectrum holes (the unoccupied subcarriers) and opportunistically get right of entry to the ones unoccupied spectrum subcarriers. Consequently, spectrum sensing or detection is an essential aspect for the implementation of CR [20].

\section{Parameters Used For 4G Network}

1. Bandwidth: Bandwidth is the difference between the top and lower frequencies in a contiguous set of frequencies. It's far generally measured in hertz, and can every now and then talk over with passband bandwidth, every now and then to baseband bandwidth, depending on context.

2. Dynamic strength management: Dynamic strength management is a layout methodology aiming at controlling overall performance and strength ranges of virtual circuits and structures, with the purpose of extending the selfsufficient operation time of battery powered systems, providing swish overall performance degradation whilst deliver power is constrained, and adapting electricity dissipation to satisfy environmental constraints.

3. Bit Error Rate: In digital transmission, the number of bit errors is the wide variety of obtained bits of a information circulate over a communique channel that has been altered due to noise, interference, distortion or bit synchronization mistakes. It's miles the variety of bit errors divided through the total range of transferred bits all through a studied time c programming language.

4. Inter Symbol Interference: In telecommunication, inter symbol interference (ISI) is a form of distortion of a signal wherein one symbol interferes with subsequent symbols. This is an unwanted phenomenon as the previous symbols have similar effect as noise, accordingly making the verbal exchange much less reliable.

5. Throughput: In communication networks, throughput or network throughput is the average rate of a success message shipping over a communique channel. This data may be introduced over a physical or logical hyperlink, or pass thru a certain network node. 
6. Access Scheme: In telecommunications and laptop networks, a channel get entry to approach or multiple get admission to approach permits numerous terminals related to the identical multi-point transmission medium to transmit over it and to proportion its capacity. Examples of shared bodily media are wi-fi networks, bus networks, ring networks, hub networks and half of-duplex factor-to-point hyperlinks. A channel-get right of entry to scheme is based totally on a multiplexing approach, which allows several statistics streams or signals to proportion the same communique channel or physical medium.

7. Delay: In a network based totally on packet switching, transmission delay is the quantity of time required to push all the packet's bits into the wire. In other words, this is the postpone due to the information-charge of the hyperlink. Transmission delay is a characteristic of the packet's length and has not anything to do with the space between the two nodes. This delay is proportional to the packet's duration in bits, it's far given with the aid of the subsequent method: DT $=\mathrm{N} / \mathrm{R}$ wherein DT is the transmission put off $\mathrm{N}$ is the wide variety of bits, and R is the price of transmission (say in bits according to second)

8. Equalization: It's Miles The Process Of Fixing The Extent Of Certain Frequencies Inside A Sign.

9. Security: Communications protection is the discipline of stopping unauthorized interceptors from getting access to telecommunications in an intelligible shape, even as nonetheless handing over content material to the intended recipients.

10. PAPR:The peak-to-average strength ratio (PAPR) is a size of a waveform, calculated from the peak amplitude of the waveform divided by the RMS value of the waveform.

\section{CONCLUSION}

In order to meet the proper desire of more than one channel get right of entry to schemes for 4G mobile wireless networks. But no matter all of the utilities of OFDMA, there are positive obstacles in using OFDM in transmission machine in contrast to its blessings.First of all OFDM sign well-known shows a totally high PAPR. Consequently, RF power amplifiers should be operated in a completely big linear region. In any other case, the sign peaks get into nonlinear area of the strength amplifier causing sign distortion. This signal distortion introduces inter modulation among the sub-carriers and out of band radiation. Accordingly, the electricity amplifiers need to be operated with massive strength again-offs. However, this leads to very inefficient amplification and high priced transmitters. Hence, it's miles rather suited to decrease the PAPR.

Secondly it's miles very touchy to frequency mistakes resulting from frequency variations between the nearby oscillators within the transmitter and the receiver. For this reason provider frequency offset reasons some of impairments inclusive of attenuation and rotation of each of the sub-carriers and ICI among sub-carriers. Inside the mobile radio environment, the relative movement between transmitter and receiver reasons Doppler frequency shifts; further, the carriers can never be flawlessly synchronized. These random frequency errors in OFDM system distort orthogonality between sub-carriers and as a consequence ICI happens. Some of strategies had been advanced to lessen this sensitivity to frequency offset.

Thirdly asynchronous records conversation offerings inclusive of internet get right of entry to are characterized by means of short communication bursts at high records fee. Few users in a base station cellular are transferring information concurrently at low steady data charge. Fourthly managing co-channel interference from nearby cells is large complex in OFDM than in CDMA. It might require dynamic channel allocation with advanced coordination among adjacent base stations.

\section{REFERENCE}

1. Chen, Y., Zhang, S., Xu, S., \& Li, G. Y. (2011). Fundamental trade-offs on green wireless networks. IEEE Communications Magazine, 49(6), 30-37.

2. Ghosh, A., Ratasuk, R., Mondal, B., Mangalvedhe, N., \& Thomas, T. (2010). LTE-advanced: next-generation wireless broadband technology. IEEE wireless communications, 17(3), 10-22.

3. Grace, D., Chen, J., Jiang, T., \& Mitchell, P. D. (2009, June). Using cognitive radio to deliver 'green'communications. In 2009 4th International Conference on Cognitive Radio Oriented Wireless Networks and Communications (pp. 1-6). IEEE.

4. Gür, G., \& Alagöz, F. (2011). Green wireless communications via cognitive dimension: an overview. IEEE network, 25(2), 50-56.

5. Yang, C., Li, J., Sheng, M., \& Liu, Q. (2012). Green heterogeneous networks: a cognitive radio idea. IET communications, 6(13), 1952-1959.

6. Devroye, N., Vu, M., \& Tarokh, V. (2008). Cognitive radio networks. IEEE Signal Processing Magazine, 25(6), 1223.

7. Goldsmith, A., Jafar, S. A., Maric, I., \& Srinivasa, S. (2009). Breaking spectrum gridlock with cognitive radios: An information theoretic perspective. Proceedings of the IEEE, 97(5), 894-914. 
8. Xing, Y., Mathur, C. N., Haleem, M. A., Chandramouli, R., \& Subbalakshmi, K. P. (2007). Dynamic spectrum access with QoS and interference temperature constraints. IEEE Transactions on mobile computing, 6(4), 423-433.

9. Pang, J. S., Scutari, G., Palomar, D. P., \& Facchinei, F. (2010). Design of cognitive radio systems under temperature-interference constraints: A variational inequality approach. IEEE Transactions on Signal Processing, 58(6), 3251-3271.

10. Shirvanimoghaddam, M., Dohler, M., \& Johnson, S. J. (2017). Massive non-orthogonal multiple access for cellular IoT: Potentials and limitations. IEEE Communications Magazine, 55(9), 55-61.

11. Shirvanimoghaddam, M., Condoluci, M., Dohler, M., \& Johnson, S. J. (2017). On the fundamental limits of random non-orthogonal multiple access in cellular massive IoT. IEEE Journal on Selected Areas in Communications, 35(10), 2238-2252.

12. Islam, S. R., Avazov, N., Dobre, O. A., \& Kwak, K. S. (2016). Power-domain non-orthogonal multiple access (NOMA) in 5G systems: Potentials and challenges. IEEE Communications Surveys \& Tutorials, 19(2), $721-742$.

13. Kiani, A., \& Ansari, N. (2018). Edge computing aware NOMA for 5G networks. IEEE Internet of Things Journal, 5(2), 1299-1306.

14. Devasis,P. (2018) Massive Mimo Technique used for 5th Generation System with Smart Antenna. International Journal of Electrical, Electronics and Data Communication, 6(7), 81-87.

15. Patil, P., Pawar, P. R., Jain, P. P., KV, M., \& Pradhan, D. (2020). Performance Analysis of Energy Detection Method in Spectrum Sensing Using Static \& Variable Threshold Level for 3G/4G/VoLTE. Saudi J Eng Technology, 5(4), 173-178.

16. Patil, P., Pawar, P. R., Jain, P. P., KV, M., \& Pradhan, D. (2020). Enhanced spectrum sensing based on Cyclostationary Feature Detection (CFD) in cognitive radio network using Fixed \& Dynamic Thresholds Levels. Saudi J Eng Technology, 5(6),271-277.

17. Jain, P. P., Pawar, P. R., Patil, P., \& Pradhan, D. (2019). Narrowband Spectrum Sensing in Cognitive Radio: Detection Methodologies. International Journal of Computer Sciences and Engineering, 7(11), E-ISSN: $2347-2693$.

18. Pardhan, D. (2017). Circular Patch With Circular Slit Patch Antenna Used For Ultra-Wide Band Application. International Journal of Electrical, Electronics and Data Communication, 5(2), 84-87.

19. Pradhan, D.(2018). Massive MIMO Technique Used For 5th Generation System With Smart Antenna. International Journal of Electrical, Electronics And Data Communication (IJEEDC), 6(7), 81-87.

20. Pardhan, D.(2019). Design of Extended Circular Patch with Rectangular Stub and Circular Slit Used For Ultra-Wide Band Application (X-Band).Journal of Applied Physics (IOSR-JAP), 11(4), 14-24.

CITATION: Shivam, Y et al (2021). A Detail Survey of Channel Access Method for Cognitive Radio Network (CRN) Applications toward 4G. South Asian Res J Eng Tech, 3(1): 31-41. 